\title{
Single and Multi Emitter Terahertz Detectors Using n-Type GaAs/AlGaAs Heterostructures
}

\author{
A. B. Weerasekara, M. B. M. \\ Rinzan, R. C. Jayasinghe, \\ S. G. Matsik, A. G. U. Perera \\ Dept. of Physics and Astronomy \\ Georgia State University \\ Atlanta, GA 30303 \\ e-mail: uperera@gsu.edu
}

\author{
M. Buchanan, H. C. Liu \\ Institute for \\ Microstructural Sciences \\ NRC Ottawa, Ont., \\ Canada K1A 0R6
}

\author{
G. von Winckel, A. Stintz, \\ S. Krishna \\ Center for High Technology Materials \\ University of \\ New Mexico, Albuquerque, NM \\ 87106
}

\begin{abstract}
Terahertz detection is demonstrated using $\mathrm{GaAs} / \mathrm{Al}_{\mathrm{x}} \mathrm{Ga}_{1-\mathrm{x}} \mathrm{As}$ n-type heterojunction interfacial work function internal photoemission (HEIWIP) detectors. A smaller workfunction $(\Delta)$ needed for terahertz detection can be achieved by using n-doped GaAs emitter and undoped $\mathrm{Al}_{\mathrm{x}} \mathrm{Ga}_{1-\mathrm{x}} \mathrm{As}$ barrier. A single emitter and a multi emitter $\mathrm{n}$-type $\mathrm{GaAs} / \mathrm{Al}_{\mathrm{x}} \mathrm{Ga}_{1-\mathrm{x}} \mathrm{As}$ HEIWIP detectors were designed, fabricated, and characterized. In both designs, $1 \times 10^{18} \mathrm{~cm}^{-3} \mathrm{n}$-type doped GaAs was used as the emitter while $\mathrm{Al}_{\mathrm{x}} \mathrm{Ga}_{1-\mathrm{x}} \mathrm{As}$ with $\mathrm{x}=\mathbf{0 . 0 4}$ for the single emitter detector and $x=0.13$ for the multi emitter detector was used as the barrier. The threshold frequency of 3.2 $\mathrm{THz}(93 \mu \mathrm{m})$ with peak responsivity of $6.5 \mathrm{~A} / \mathrm{W}$ at $7.1 \mathrm{THz}$ at $6 \mathrm{~K}$ was successfully demonstrated for the single emitter detector while $5 \mathrm{THz}(60 \mu \mathrm{m})$ threshold frequency and 0.32 A/W peak responsivity was observed for the multi emitter detector at $5 \mathrm{~K}$. In addition, the peak quantum efficiency of $19 \%$ and peak detectivity of $\sim 5.5 \times 10^{8}$ Jones under a bias field of $0.7 \mathrm{kV} / \mathrm{cm}$ at $6 \mathrm{~K}$ were obtained for the single emitter detector.
\end{abstract}

\section{INTRODUCTION}

Terahertz region, which is relatively less explored in the electromagnetic spectrum, lies between $300 \mathrm{GHz}$ and 30 terahertz in the electromagnetic spectrum. In recent years, attention was drawn beyond the infrared region into terahertz region. There is an enormous potential for applications in this spectral range such as medical diagnostic, pharmaceutical, security, wireless communications, and astronomy $[1,2]$. However, the main challenge in this field is the lack of suitable terahertz sources and detectors. Thermal detectors that are used in this region are slow in response and are difficult to integrate into focal plane arrays for imaging. Therefore, photon detectors which are faster and easy to integrate into focal plane arrays are good candidates for terahertz applications[3].

In HEIWIP structures, an undoped alloy semiconductor material is used as the barrier and heavily doped semiconductor as the emitter. The internal workfunction, $\Delta$ is defined from the top of the Fermi energy level $\left(E_{F}\right)$ in the

This work was supported in part by the U. S. NSF under grant No. ECS0553051 . emitter to the bottom of the conduction band level of the barrier and is given by $\Delta=\Delta_{\mathrm{x}}+\Delta_{\text {narr }}-\mathrm{E}_{\mathrm{F}}$. Here $\Delta_{\mathrm{x}}$ is the conduction band offset between the emitter and the barrier due to Al composition (x), $\Delta$ narr is the conduction band narrowing in the emitter layer due to doping, and $\mathrm{E}_{\mathrm{F}}$ is the Fremi energy with respect to the bottom of the emitter conduction band. Threshold frequency, $f_{0}$ is given by $f_{0}=\Delta$ $/ 4.133$ in terahertz. Here $\Delta$ is in meV. The threshold frequency of the detector $\left(f_{0}\right)$ can be tailored by changing the alloy fraction $(\mathrm{x})[4,5]$.

In general, designing p-type HEIWIP detectors is easier because of the smaller Fermi level shift due to p-doping when compared with n-doping. Therefore, previously reported results on HEIWIP detectors are limited to p-type structures[5-8]. In n-type, the fermi level changes drastically even for smaller change in n-doping due to the smaller effective mass of electron. Because of this, when designing smaller workfunctions $(\Delta)$ for terahertz detectors, accuracy of doping should be very precise in order to get results consistent with designs. p-type HEIWIP detectors have shown the ability to push the threshold beyond $5 \mathrm{THz}(>60$ $\mu \mathrm{m})$ limit $[4,6,9]$. Tailorability of threshold frequency $f_{0}$ can be achieved by changing $\mathrm{Al}$ fractions, and has been demonstrated for p-type HEIWIP terahertz detectors with different $\mathrm{Al}$ fractions resulting in $f_{0}=4.6,3.6$, and $3.2 \mathrm{THz}$ threshold frequencies[4]. The Al fraction used for the 3.2 $\mathrm{THz}$ threshold detector is 0.005 and is close to the practical lower limit for MBE growth. It is not possible to lower the workfunction $(\Delta)$ by increasing the emitter doping in p-type HIEWIP since Fermi level will not reach the required level before GaAs becomes metallic as p-doping increases. Therefore, lowering the workfunction $(\Delta)$ further may not be possible in p-type HEIWIP devices. If $\mathrm{p}$-doped $\mathrm{Al}_{\mathrm{x}} \mathrm{Ga}_{1-\mathrm{x}} \mathrm{As}$ is used as the emitters and undoped GaAs as a barrier (inverted structure), workfunction $(\Delta)$ can be further reduced by increasing the $\mathrm{Al}$ fraction and thereby a lower threshold frequency. However, the reported lowest threshold obtained with inverted structure is $2.3 \mathrm{THz}(128 \mu \mathrm{m})$. The other alternative is to use n-type GaAs emitters in HEIWIP detectors. For the same $\mathrm{Al}$ fraction composition and the same 

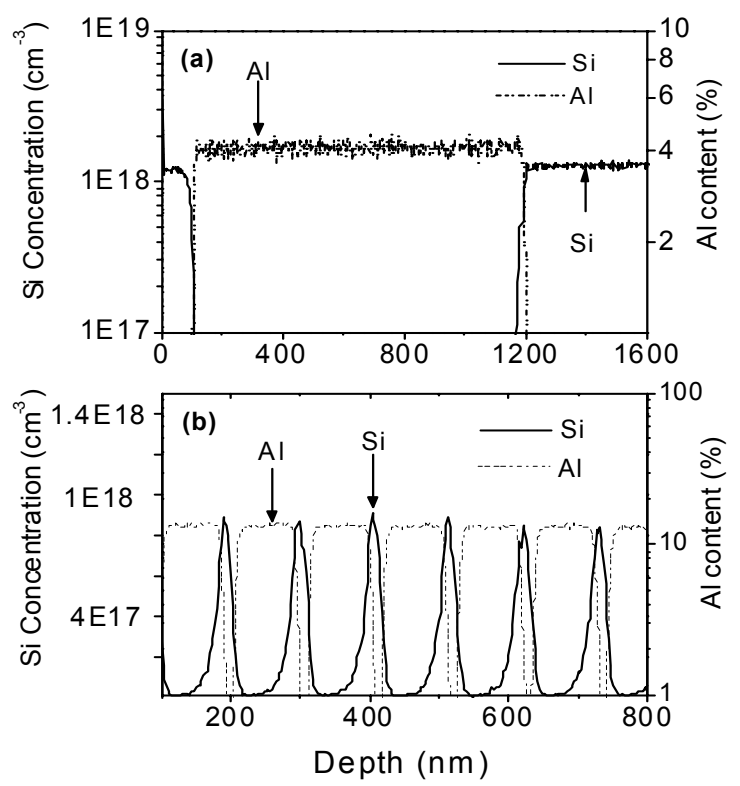

Fig 1: (a) SIMS data of single barrier n-type HEIWIP structure. Top and bottom contact layers (emitters) are n-doped to $1 \times 10^{18} \mathrm{~cm}^{-3}$. Aluminum fraction in the barrier is $4 \%$ (b) SIMS data of the multi emitter structure. Only seven periods are shown for clarity. The aluminum fraction is about $13 \%$ $\mathrm{cm}^{-3}$ and Si doping is $\sim 1 \times 10^{18} \mathrm{~cm}^{-3}$.

emitter doping concentration, the workfunction in n-type HEIWIP is smaller than that of p-type HEIWIP due to the lower effective mass of electrons compared to the effective mass of holes. According to initial theoretical calculations, it is possible to achieve a threshold frequency below $3 \mathrm{THz}$ in an n-type HEIWIP with a relatively larger Al fraction. For an example, $1 \mathrm{THz}$ threshold frequency can be achievable in ntype HEIWIP with an Al fraction of $0.03(3.0 \%)$ which is possible in thin film growth techniques. Here, the capability of extending the zero response threshold $\left(\mathrm{f}_{0}\right)$ to low terahertz region using n-type emitters in HEIWIP is experimentally demonstrated.

\section{EXPERIMENT}

\section{A. Device Designing}

Two HEIWIP (A single emitter and a multi emitter) detector structures were designed to test the performance of n-doped GaAs HEIWIP detectors. The single barrier device structure consists of an undoped $1 \mu \mathrm{m}$ thick $\mathrm{Al}_{\mathrm{x}} \mathrm{Ga}_{1-\mathrm{x}} \mathrm{As}$ $(\mathrm{x}=0.04)$ barrier layer sandwiched between two n-doped (Si) $1 \times 10^{18} \mathrm{~cm}^{-3} \mathrm{GaAs}$ contact layers with the top contact being $100 \mathrm{~nm}$ and the bottom contact being $700 \mathrm{~nm}$ in thickness. GaAs n-doped to $5 \times 10^{18} \mathrm{~cm}^{-3}$ with $\mathrm{Si}$ was used as the substrate to enhance the light reflection from the substrate. The top and the bottom contact layers work as emitters for reverse and forward bias operations. The $\mathrm{Si}$ doping concentration and the $\mathrm{Al}$ fraction were verified by secondary ion mass spectrometry (SIMS) as shown in Fig. 1 (a). The thickness of the top contact layer was kept to $100 \mathrm{~nm}$ to allow a substantial amount of light to pass through to the bottom contact. The multi emitter device consists of twelve periods of $1 \times 10^{18} \mathrm{~cm}^{-3} \mathrm{n}$-doped $20 \mathrm{~nm}$ thick GaAs emitter and undoped $80 \mathrm{~nm}$ thick $\mathrm{Al}_{0.13} \mathrm{Ga}_{0.87} \mathrm{As}$ barrier. The top and bottom contact layers were $1 \times 10^{18} \mathrm{~cm}^{-3}$ n-doped with 100 and $700 \mathrm{~nm}$ thicknesses respectively and $5 \times 10^{18} \mathrm{~cm}^{-3} \mathrm{n}-$ doped GaAs was used as the substrate. NiGeAu was deposited on the bottom and the top layers as ring ohmic contacts to make electrical contacts. Note that the highly doped substrate is electrically isolated from the active layers.

\section{B. Device Characterization}

\section{1) Single Emiter device}

The electron gas in the single emitter structure should be treated as a 3D distribution. The calculated Fermi energy in the emitter layers of the single barrier device, $E_{F}$ is $55 \mathrm{meV}$ while the conduction band discontinuity in the GaAs/AlGaAs interface is $32 \mathrm{meV}$ for $\mathrm{x}=0.04$. Considering band gap narrowing [10] of $35-45 \mathrm{meV}$ in the GaAs emitter layer due to $1 \times 10^{-18} \mathrm{~cm}^{-3} \mathrm{n}$ - doping, the calculated workfunction $(\Delta)$ of the single barrier device is between $\sim 10-20 \mathrm{meV}$, which corresponds to $2.4-5.0 \mathrm{THz}$ (125 -60 $\mu \mathrm{m}$ ) cutoff frequency. Dark current-voltage measurements were performed on the devices from 4.2 to $120 \mathrm{~K}$ to obtain workfunction $(\Delta)$ using Arrhenius analysis. According the estimated workfunction using Arrhenius analysis is $13-14 \mathrm{meV}(88-95 \mu \mathrm{m})$ for the single emitter device. Spectral measurements at $6 \mathrm{~K}$ were performed using a fast fourier transform infrared spectrometer (FTIR) and a silicon composite bolometer was used to calibrate the raw

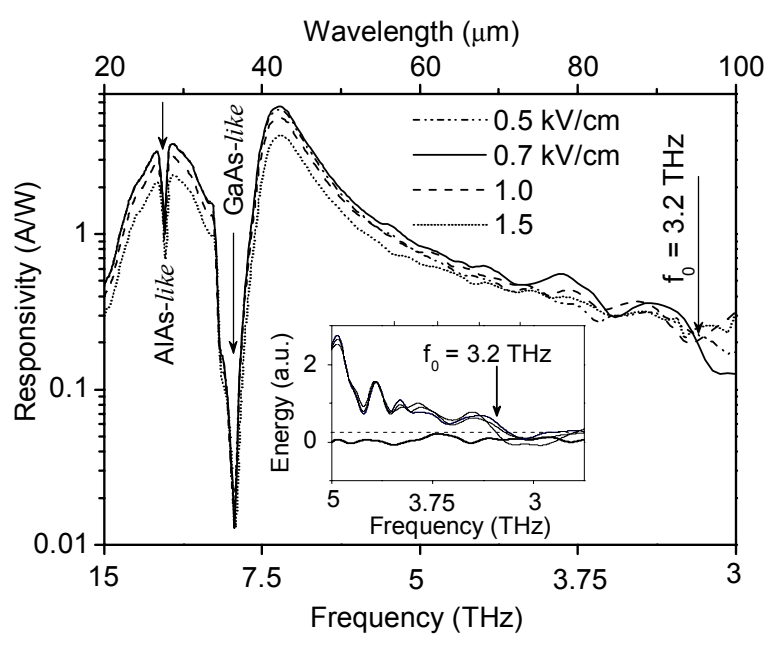

Fig 2: Spectral response for single emitter device at $6 \mathrm{~K}$. The threshold frequency of $3.2 \mathrm{THz}(93 \mu \mathrm{m})$ was determined by considering the instrument noise level as shown in the inset. The peak response was found to be $6.5 \mathrm{~A} / \mathrm{W}$ at $7.1 \mathrm{THz}$. Two narrow dips are due to the GaAs and AlAs like phonons. 
spectra. The measured spectral responses for different bias fields are shown in Fig. 2. The zero response threshold frequency, which is found to be $3.2 \mathrm{THz}(93 \mu \mathrm{m})$, was estimated by considering instrument noise level. The maximum peak response of $6.5 \mathrm{~A} / \mathrm{W}$ at $7.1 \mathrm{THz}(42 \mu \mathrm{m})$ was obtained at $0.7 \mathrm{kV} / \mathrm{cm}$ bias field. A strong light reflection occurs around $11 \mathrm{THz}(27 \mu \mathrm{m})$ and $8.3 \mathrm{THz}(36$ $\mu \mathrm{m})$ due to the AlAs-like and GaAs optical phonons, giving two minima around $11 \mathrm{THz}(27 \mu \mathrm{m})$ and $8.3 \mathrm{Thz}(36 \mu \mathrm{m})$ in the responsivity spectra. Up to now, the lowest threshold frequency reported for HEIWIP detectors is $2.3 \mathrm{THz}(128$ $\mu \mathrm{m})[6]$. It is noteworthy that the response of the single barrier detector reported here matches the 10 emitter p-type detector reported in ref.[6]. The peak values of responsivity $\left(R_{P}\right)$, quantum efficiency $\left(\eta_{p}\right)$, and detectivity $\left(D_{p}{ }^{*}\right)$ at 7.1 $\mathrm{THz}$ for different bias fields are given in Table I. The detectivity was calculated using the shot noise. The device response decreases with temperature and vanishes after 25 $\mathrm{K}$. The device can also be operated under reverse bias and $\mathrm{R}_{\mathrm{p}}$ of $1.7,1.1$, and $0.7 \mathrm{~A} / \mathrm{W}$ at $10 \mathrm{THz}(30 \mu \mathrm{m})$ were observed at $-0.25,-0.5$, and $-0.75 \mathrm{kV} / \mathrm{cm}$ bias respectively.

TABLE I.

The peak responsivity (Rpeak), peak quantum efficiency ( $\eta$ ), and detectivity $\left(\mathrm{D}^{*}\right)$ are given at different applied bias fields for the single emitter detector.

\begin{tabular}{|c|c|c|c|}
\hline $\begin{array}{c}\text { Bias Field } \\
(\mathrm{kV} / \mathrm{cm})\end{array}$ & $\begin{array}{c}\mathrm{R}_{\text {peak }} \\
(\mathrm{A} / \mathrm{W})\end{array}$ & $\begin{array}{c}\eta \\
(\%)\end{array}$ & $\begin{array}{c}\mathrm{D}^{*} \\
(\text { Jones })\end{array}$ \\
\hline 0.2 & 3.0 & 8.9 & 5.5 \\
\hline 0.5 & 6.1 & 18.2 & 6.4 \\
\hline 0.7 & 6.5 & 18.9 & 5.5 \\
\hline 1.0 & 5.5 & 16.5 & 3.4 \\
\hline 1.5 & 4.1 & 12.3 & 2.1 \\
\hline
\end{tabular}

\section{2) Multi Emitter Device}

Spectral response measurements were carried out for the multi layer device as in the single emitter device case. The calculated workfucntion $(\Delta)$ for the multi emitter device must be larger than $60 \mathrm{meV}$ which corresponds to a threshold shorter than $20 \mu \mathrm{m}$. However, a workfunction $(\Delta)$ of $\sim 22 \mathrm{meV}(\sim 5 \mathrm{THz})$ was obtained from Arrhenius analysis. The threshold frequency obtained from the spectral measurements is $\sim 5 \mathrm{THz}(20 \mathrm{meV})$ as shown in Fig. 3 and is in close agreement with the arrhenius analysis. According to the SIMS measurements, a considerable amount of doping migration into the barrier layer is visible. This doping migration can change the Fermi level alignment from the expected situation across the interface causing the effective barrier height much smaller. It should be mentioned that further studies are needed to understand how this discrepancy occurs in the multilayer detector. Furthermore, the spectral responsivity of the multi emitter detector is lower than the single emitter device and can be

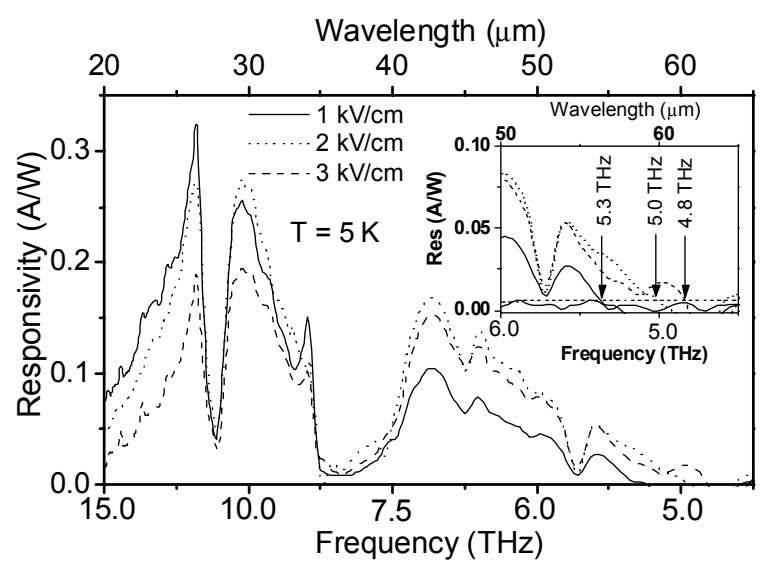

Fig 3: The responsivity of multi emitter device with three different applied bias fields at $5 \mathrm{~K}$. The maximum responsivity of $0.32 \mathrm{~A} / \mathrm{W}$ at $11 \mathrm{THz}$ at the bias filed of $1 \mathrm{kV} / \mathrm{cm}$ is obtained. Threshold frequencies of $5.3,5.0$, and

$4.8 \mathrm{THz}$ were estimated for bias fields of $1.0,2.0$, and $3.0 \mathrm{kV} / \mathrm{cm}$ respectively using the system noise level (see the inset)

attributed to the doping migration in the multi emitter device. Due to the doping migration, density of states can be less leading to low photoabsorption efficiency.

\section{PHOTORESPONSE MODELING}

Spectral responsivity for HEIWIP detectors can be carried out as discussed in Ref.[5,11]. The photo responsivity $\mathrm{R}$ can be given as in Eq. [1].

$$
R(\lambda)=\frac{q \eta \lambda}{h c}
$$

Where, $q$ is the electron charge, $\eta$ is the total quantum efficiency, $h$ is the Plank constant, and $c$ is the speed of light. Total quantum efficiency depends on the light absorption efficiency in the emitter layer $\left(\eta_{\mathrm{a}}\right)$, photoexcited carrier emission efficiency $\left(\eta_{\mathrm{e}}\right)$ over the workfunction $(\Delta)$, and the carrier collection efficiency $\left(\eta_{c}\right.$. $)$ which can be considered as unity in most cases. The absorption efficiency $\left(\eta_{\mathrm{a}}\right)$ can be calculated using the free carrier absorption mechanism[5]. Photoexcited carrier emission efficiency $\left(\left(\eta_{\mathrm{e}}\right)\right.$ can be calculated using the escape cone model [11, 12] with or without considering phonon emission. The spectral response including phonon emission was modeled for the single emitter device and is shown in Fig. 4. Model calculation matches well in the lower frequency range $(<10 \mathrm{THz})$. There is a small discrepancy between the calculated and the experimental spectra above $11 \mathrm{THz}$. This discrepancy may be due to energy independent scattering length assumption.

\section{DISCUSSION AND CONCLUSION}

In this work, n-type GaAs/AlGaAs HEIWIP terahertz detectors have been successfully demonstrated. When compared with p-type HEIWIP detectors, a smaller threshold frequency can be obtained with a relatively higher $\mathrm{Al}$ 
fraction. Thereby, n-type HEIWIP devices are favorable for terahertz detection. An Al fraction of 0.005 , which is around the lowest limit for MBE, was used to obtain $3.2 \mathrm{THz}$ threshold in a p-type HEIWIP detector[4] while the same threshold frequency can be obtained with Al fraction of 0.04 in the n-type HEIWIP detector as shown in this study. Ability to design smaller workfunction with higher Al fractions in n-type GaAs is favorable for growth. Extending the threshold frequency below $3 \mathrm{THz}$ in photon detectors is possible. In addition, successful modeling for n-type GaAs/AlGaAs HEIWIP devices was also carried out.

\section{REFERENCES}

[1] P. H. Siegel, "Terahertz Technology," IEEE Transaction on Microwave Theory and Techniques ol. 50, pp. 910-928, 2002.

[2] P. H. Siegel, "Terahertz technology in biology and medicine," Microwave Theory and Techniques, IEEE Transactions on, vol. 52, pp. 2438-2447, 2004.

[3] A. Rogalski, "Infrared detectors: status and trends," Progress in Quantum Electronics, vol. 27, pp. 59210, 2003.

[4] S. G. Matsik, M. B. M. Rinzan, A. G. U. Perera, H. C. Liu, Z. R. Wasilewski, and M. Buchanan, "Cutoff tailorability of heterojunction terahertz detectors," Appl. Phys. Lett., vol. 82, pp. 139-141, 2003.

[5] D. G. Esaev, M. B. M. Rinzan, S. G. Matsik, and A. G. U. Perera, "Design and optimization of $\mathrm{GaAs} / \mathrm{AlGaAs}$ heterojunction infrared detectors," $J$. Appl. Phys., vol. 96, pp. 4588-4597, 2004.

[6] M. B. M. Rinzan, A. G. U. Perera, S. G. Matsik, H. C. Liu, Z. R. Wasilewski, and M. Buchanan, "AlGaAs emitter/GaAs barrier terahertz detector with a $2.3 \mathrm{THz}$ threshold," Appl. Phys. Lett., vol. 86, pp. 071112, 2005.

[7] S. G. Matsik, M. B. M. Rinzan, D. G. Esaev, A. G. U. Perera, H. C. Liu, and M. Buchanan, "20 $\mu \mathrm{m}$ cutoff heterojunction interfacial work function internal photoemission detectors," Appl. Phys. Lett., vol. 84, pp. 3435-3437, 2004.

[8] D. G. Esaev, S. G. Matsik, M. B. M. Rinzan, A. G. U. Perera, H. C. Liu, Z. R. Wasilewski, and M. Buchanan, "Resonant Cavity Enhanced GaAs/AlGaAs IR Detectors," SPIE Proc., vol. 4999, pp. 467-477, 2003.

[9] A. G. U. Perera, S. G. Matsik, M. B. M. Rinzan, A. Weerasekara, M. Alevli, H. C. Liu, M. Buchanan, B. Zvonkov, and V. Gavrilenko, "The Effects of Light-Heavy Hole Transitions on the Cutoff Wavelengths of Far Infrared Detectors," Infrared Phys. and Tech., vol. 44, pp. 347-353, 2003.

[10] H. Yao and A. Compaan, "Plasmons, photoluminescence, and band-gap narrowing in very heavily doped n-GaAs," Applied Physics Letters, vol. 57, pp. 147-149, 1990.
[11] A. G. U. Perera, H. X. Yuan, and M. H. Francombe, "Homojunction internal photoemission far-infrared detectors: Photoresponse performance analysis," $J$. Appl. Phys., vol. 77, pp. 915-924, 1995.

[12] J. M. Mooney and J. Silverman, "The theory of hotelectron photoemission in Schottky-barrier IR detectors," IEEE Trans. Electron Devices, vol. 32, pp. 33-39, 1985. 